\title{
Nutritional and Biochemical Composition of Amla (Emblica officinalis) and its Therapeutic Impact: A Review
}

\author{
Ali Ikram ${ }^{1}$, Waseem Khalid ${ }^{1 *}$, Maryam Aziz ${ }^{1}$, Muhammad Adnan Arif ${ }^{2}$, \\ Ravi Prakash Jha ${ }^{3}$, Muhammad Zubair Khalid ${ }^{1}$, Chasheen Fizza ${ }^{1}$, \\ Muhammad Zarnoor Mehmood ${ }^{1}$, Muhammad Haseeb ${ }^{1}$, Muhammad \\ Abdul Rahim ${ }^{1}$, Sadia Naeem ${ }^{1}$, Fatima Sultana ${ }^{1}$ \\ ${ }^{1}$ Institute of Home and Food Sciences, Government College University, Faisalabad, \\ Pakistan \\ ${ }^{2}$ National Institute of Food Science and Technology, University of Agricultural, \\ Faisalabad, Pakistan \\ ${ }^{3}$ Department of Community Medicine, Institute of Medical Sciences, Banaras Hindu \\ University, Varanasi, India \\ *Corresponding Author: Waseem Khalid, Institute of Home and Food Sciences, \\ Government College University, Faisalabad, Pakistan.
}

Received: December 25, 2020

Published: January 28, 2021

(C) All rights are reserved by Waseem Khalid., et al.

\begin{abstract}
From ancient times, plants played a vital role in the development of mankind and was an exceptional source of natural medicine. The difficulty of the production of chemical medications, their side effects on safety, and the expense of rebellion have led researchers worldwide to concentrate on therapeutic plant science. Pakistan has a large collection of various plant species, with around 5,000 plants claiming to have important medicinal values. The papers published in recent decades on medicinal plants relate to the function of various plant bioactive composites commonly used in the cure of numerous human diseases. Bioactive compounds such as ascorbic acids, flavonoids, tannins, terpenoids, saponins, and several other components are reported to have been verified to have various medicinal activities, such as anti-inflammatory, antioxidant, antimicrobial, radiological, antitisinous, hepatoprotective, immune-modulatory, hypolipidemic and several other actions. The drug also has anti-cancer, antidepressant, antidiabetic, injury healing, antiulcerogenic, and so on. The present review article sums up the phytochemical components and pharmacology of the Emblica officinalis plant and its traditional application. Amla phyto-chemicals, including ellagic acid, emblicanine A, emblacani B, Gallic acid, phyllantine, quercetin and phyllantidine were found to have various biological activities such as antioxidants, antimicrobials, antiinlammative, antidiabetic, anti-radiation protection, chemopreventive and healing of wounds.
\end{abstract}

Keywords: Amla; Applications; Traditional Uses and Medicinal Effects

\section{Introduction}

Emblica officinalis (EO) holds a sacred place in Ayurveda, an Indian indigenous medicine system. EO is the first tree grown in the world, according to Indian mythology. The other names of this tree are Amla, Indian Glasberry, or Phyllanthus emblica. It belongs to the family Euphorbiaceae. It is observed that intuitive species of
Indian amla grow in both tropical and subtropical zones of China, Pakistan, Srilanka, South Asia, and Malaysia. The plant, leaves and fruit of amla have been listed in the figure 1. Aryuyeda believed that the fruit of EO increase defense against numerous diseases such as cancer, ulcer, anemia, heart diseases, liver, and diabetes, for that reason they extensively used EO fruits. It also acts as an antioxi- 
dant, antitussive, gastroprotective, immunomodulatory, analgesic, and antipyretic. Besides, it is useful to control the cholesterol level and also boost memory in ophthalmic disorders. It is also beneficial in snake venom neutralization and as an antimicrobial.

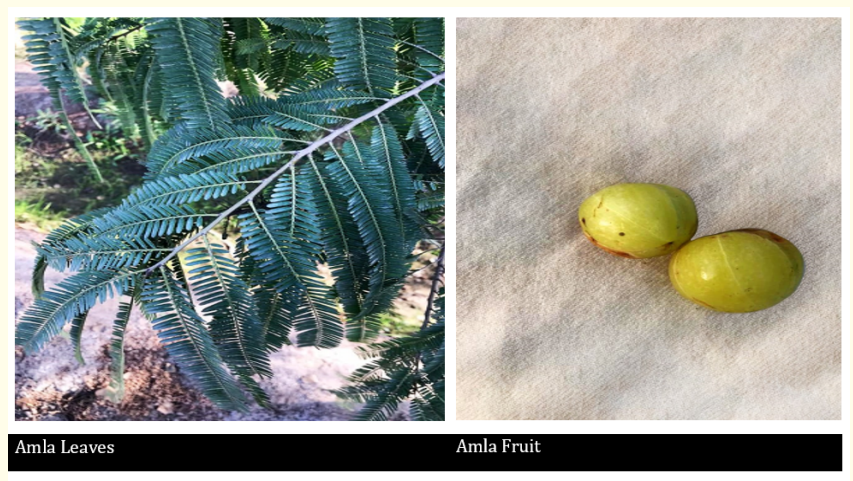

Figure 1: Amla Leaves and Amla Fruit.

\section{Traditional uses}

Amla considered a powerful Rejuvenator and valuable in delaying the senescence and degenerative process. Chinese, Sir Lankan, Siddha, and Unani Tibetan utilize E. Officinalis for medicine purposes. This helps improve lifespan, digestion, and relieve constipation. According to the Ayurvedic Medicine System EO significantly decreases fever, inflammation, blood cleansing, heart health improving, benefits the vision, stimulates hair growth, strengthens the body, relieves asthma, and improves the intellect ability. Fruits that are astringent used in many folk medicines for the treatment of ophthalmic disorders, hyperacidity, osteoporosis, gastritis, premature hair graying, dyspepsia, colitis, hemorrhoids, constipation, cough, anemia, hematuria, diabetes, asthma, and tiredness. E. officinalis probed to have anti-inflammatory, diuretic, laxative, cardioprotective, antipyretic, and hepatoprotective properties. EO also reported as a hair tonic and digestive medicine, also effective against peptic ulcer [1].

\section{Chemical constituents of Emblica officinalis}

It's challenging for drug industries to recognize the exact genotype of medical plants. Precincts of morphological and techniques to authentication have to need to create the latest protocols in botanical quality control. A DNA-based marker for EO and the Random Polymorphic Amplified DNA (RAPD) technique has been developed to identify putative markers (1.1 kb) unique to EO recognition. Furthermore, in commercial samples of EO SCAR (sequence characterized amplified region) marker is considered to be valuable for the identification, and the SCAR marker is generated through using the RAPD application [2]. Phenolic compounds, amino acids, tannins, alkaloids, and carbohydrates are the major components of the EO. The highest level of vitamin $C$ in fruit juice is (478.56 mg/100 ml) and when mixed with other fruit, the fruit improved its nutritional profile with vitamin C content [3]. Many types of beneficial compounds such as quercetin, ellagic acid, gallic acid, corilagin, chebulinic acid, 1,6-di-O-galloyl beta-D glucose, 3,6-di-0-galloyl-glucose, 3 ethylgallic acid (3 ethoxy 4,5 dihydroxy benzoic acid), 1-0-galloyl-beta-D-glucose, chebulagic acid, and isostrictiniin, isolated from it. In addition to, also possess some flavonoids including, kaempferol 30 alpha L (6" ethyl) kaempferol and rhamnopyranoside 30 alpha L ( 6 "methyl) rhamnopyranoside [4]. Similarly, a new acylated apigenin glucoside (apigenin 70 (6 "butyryl beta glucopyranoside), along with the known compounds gallic acid, 1,2,3,4,6-penta-0-galloylglucose, methyl gallate, and luteolin-4'-Oneohesperiodoside were also identified after the isolation from the methanol extract of the Phyllanthus emblica leaves [5]. A number of chemical components found in amla are listed in table 1.

\begin{tabular}{|l|c|c|}
\hline $\begin{array}{c}\text { Fruit pulp (Kumar., } \\
\text { et al. 2012) }\end{array}$ & $\begin{array}{c}\text { Leaves (Singh., } \\
\text { et al. 2011) }\end{array}$ & $\begin{array}{c}\text { Seed (Khan, } \\
\text { 2009) }\end{array}$ \\
\hline Moisture & Gallic Acid & Fixed Oil \\
\hline Mineral & Chebulic Acid & Phosphatides \\
\hline Crude Cellulose & Ellagic Acid & Essential oil \\
\hline Albumin & Chebulinic Acid & \\
\hline Gum & Amlic Acid & \\
\hline Tannin & $\begin{array}{c}\text { Alkaloids } \\
\text { Phyllantine }\end{array}$ & \\
\hline Gallic Acid & Phyllantidine & \\
\hline
\end{tabular}

Table 1: Chemical Constituent from Different Plant Parts of Amla.

\section{Phytochemical}

In recent years, many scientific kinds of literature have recorded different Phyto-constituents of E. officinalis. The amla fruit includes numerous bioactive components including isostrictiniin, ellagic acid, apigenin, chebulinic acid, quercetin, gallic acid, chebulagic acid. The tannins also found in the fruit extract of amla arepedun- 
culagin, emlicanin A, phyllaemblicin B, emblicanin B and punigluconine $100 \mathrm{~g}$ of edible fruit have been reported to be $470-680 \mathrm{mg}$ of Vit. C. In ethanolic extracts of amla [9], recently identified Quercetin and b-sitosterol. Another study confirmed the existence of 5 major plants, including 5-hydroxymethylfurfural, 1, 2, 3-benzenetriol (synonym: Pyragallol), 2-acetyl-5-methylfuran. Recently two new chalconoid analogues, emblirol B [3] and emblirol A [2], have been isolated from the roots of embilica with a molecular formula C19H2406 [3]. The discovery of various phytochemicals in different sections of amla is very significant and understand its therapeutic role, along with mechanistic action in the attack against different disorders. Also, known phytocompounds can be docked for the understanding of their goals and related therapeutic activities.

\section{Applications of Emblica officinalis}

Role in cancer treatment

Earlier Triphala was considered to reveal some prospective for chemoprevention. The benzo-a-pyrene (B-a-P) responsible for papilomagenesis incidence in the fore-stomach of mice could be reduced through the addition of Triphala in the diet. It has become more active in decreasing tumor incidences relative to its components. The antioxidant level of animals which would have led to chemoprevention also increased substantially with Triphala [10]. Breast cancer considered one of the most communal cancers among females. Major factors associated with breast cancer were lipid metabolism enzymes, fats, and lipoproteins. Kalpaamruthaa (KA) is a improved preparation of Siddha which contains EO, honey and Semecarpus Anacardium. As treated by KA and SA, the raised levels of phospholipids, free fatty acids triglycerides, total cholesterol, free cholesterol, and the replacement of ester cholesterol in blood, liver, and kidney in animals with cancer have reversed to almost normal rates [11].

Chemoprevention with food phytochemicals is generally recognized to be one of the most vital cancer control methods. EO is known for its special flavonoids and tannins, which reveal very strong antioxidants properties. In Swiss albino mice, the inhibition of tumor incidence rate from this plant's fruit extract was tested on a two-stage cycle of skin carcinogenesis. Chemopreventive potential of the EO fruit extract was found on skin tumorigenesis induced by 7,12-dimethylbenz(a)anthracene (DMBA) in Swiss albino mice [12]. A significant polyphenol, gallic acid, observed in Triphala responsible for the reduction of cancer cell growth [13]. Experimen- tally, EO ethanoic extract was probed for protection against DMBA induced genotoxicity. Before single intraperitoneal DMBA injection different concentrations $(100,250,500 \mathrm{mg} / \mathrm{kg})$ of EO fruit administered orally to Swiss albino mice for seven consecutive days which significantly reduced the frequency of bone marrow micronuclei. The anti-oxidant ability and modulative impact on detoxification of enzymes and hepatic activation are the main factors in EO's defense system [14].

\section{Diabetic uses}

Blood sugar levels fell significantly in healthy rats and alloxan (120 mg/kg) after oral administration (100 mg/kg body weight) within 4 hours. Production of diabetic cataract substantially decreased by EO and a supplemented fraction of its tannins in rats [15]. Aldose reductase (AR) engages in the growth of complications of secondary diabetes comprising cataracts. EO as a verified inhibitor of Aldose reductase. A significant approach to managing diabetic problems would be to examine the medicinal advantages of natural ingredients which would incorporate people into their everyday lives [16].

\section{Impacts on the liver}

Amla fruits reportedly have been used in Ayurveda [17], for hepatoprotection, and Phyllanthus emblica extract has been tested for hepatic rat damage due to ethanol [18]. The extent of hepatic fibrosis caused by thioacetamide and carbon tetrachloride was lessened by a hydroalcoholic (50\%) extract of EO fruit (EO-50). Because of its antioxidant function, EO-50 effectively reverting profibrogenic events. EO-50 probed hepatoprotective role in antituberculosis drug-induced hepatic injury. Due to the perceived character of antioxidant, membrane stabilizer, and inhibitory CYP2E1, the E0-50 has hepatic protection activity [19]. Liver toxicity in Wistar rats also inhibited by EO [20]. EO and Chyayanaprash hepatoprotective functions were reviewed in rats with CCL causing liver damage. The extracts revealing reduced liver and serum peroxide, alkaline phosphatase (ALP), and glutamate-pyruvate transaminase (GPT) have been reported to suppress hepatotoxicity triggered by acute as well as chronic $\mathrm{CCl}_{4}$ administration extract. The use of $\mathrm{CCl}_{4}$ also induces hepatic fibrosis as revealed with elevated levels of pathological and collagen-hydroxyproline testing. The two extracts have been reported to significantly hinder these increased levels, suggesting that extracts can decrease the induction of fibrosis in rats [21]. 
The cardio-protective activity

The chronic effects of Amla's homogeneous and fresh fruit as oral administration on myocardial system antioxidant and ischemic-reperfusion injury (IRI) to the oxidative stress was tested in mice. Prolonged administration stimulates myocardial adaptation through swelling endogenous antioxidants as well as secures the heart of rats against IRI-associated oxidative stress [22].

\section{Anti-ulcers characters}

A herbomineral Ayurvedic formulation called Pepticare has been analyzed for its anti-ulcer and antioxidants in rats consisting of EO, Tinospora cordifolia, and Glycyrrhiza glabra. Peptic has antiulcer attitudes, due to its antioxidant properties [22]. EO extract (EOE) was tested for the treatment of ulcers. EOE has powerful curative and therapeutic impacts on the ulcer, which can affect both defense and offensive mucosal factors [23].

\section{Antioxidant activities}

Because of behavioral and biochemical abnormality changes due to cold stress, a study on EO has been carried out. The oral dispensation of Triphala by approximately one $\mathrm{g} / \mathrm{kg}$ of the animal body until the 48-day period prevented cool stress-induced behavioral and biochemical abnormalities of albino rats. Hence the deemed shielding drug against stress is Triphala supplement [24]. In the aging rats, accelerated urea-nitrogen status is also suppressed by feeding Amla or Sun Amla ethyl acetate extract (EtoAc). Conspicuous lessening of thiobibeutric acid-reactive elements volumes of serum, renal homogenate and mitochondria in old age mice are due to the oral intake of this extract. Thus urging that oxidative stress undergoing would be ameliorated by Amla. Moreover, EtoAc extracts from Amla or sun Amla extracts have substantially prevented up-grades in the COX-2 aorta of aged mice of nitric oxide synthase (iNOS) and cyclooxygenase. Restricting the NF-KappaB activation in the aged rats, the expression levels of COX-2 and iNOS are leveled down by the EtoAc extracts of Amla or Sun Amla. Ultimately for the prevention of age-associated renal ailments, Amla will come to be a very fruitful antioxidant [25]. Chashan the increase in renal gamma-glutamyl transpeptidase (GGT) activity triggered via hexachlorocyclohexane $(\mathrm{HCH})$ was declined by pre-feeding Amla. Amla feeding was recognized to be an excellent part of a hepatic antioxidant mechanism and to deplete cytotoxic products which could be caressed by feeding HCH [26]. The rat's gut revealed for the entire body gamma radiation (WBI) was reported to have strengthened the activity of xanthine oxidoreductase and decreased superoxide dismutase activity, However, the amount of sham irradiation was retrieved for 7 days when animals were treated with Triphala. Administration with Triphala recommended as the prevention from oxidative detriment caused by the exposure of the whole body to the radiation. The whole body of irradiated mice was shielded by Triphala. Protection was conciliated through repression of oxidative damage in cells and organs. The potential to thrive into a novel herbal radioprotector for practical application is signaled towards this drug [27]. Sedimentary extracts of the Terminalia chebula, EO, and Terminalia belerica supplies and their combination of Triphala equi-portional were tested for their in vitro antioxidant activities. The gamma-radiation promoting a strand break in plasmid DNA (pBR322) was considerably dwindled by the Triphala and its constituents. Terminalia chebula 's increased scavenging activities while its higher capability in lipid peroxidation and plasmid DNA testing is demonstrated by the EO, and their mixture Triphala is reckoned as more effective because of the incorporate activity of every single constituent [28].

\section{Antipyretic and analgesic behavior}

The extracts of EO are strengthened by strong antipyretic and analgesic action. There has been a substantial decrease in brewer's yeast hyperthermia in rats with just a single dose of ethanol extracts and aqueous extract $(500 \mathrm{mg} / \mathrm{kg})$. Prominent inhibitory effects on acetic acid-induced writhing retort in mice were reduced by both of these extracts was revealed in analgesic test [29]. Such symptoms may have been caused by the involvement of alkaloids, tannins, amino acids, phenolic compounds, or carbohydrates.

\section{Cytoprotective, gastroprotective and antitussive properties}

Amla has been held responsible for its cytoprotective, chromium-induced immuno-modulatory effects of oxidative vandalism. Using the process of macrophage and phagocytosis gamma-IFN production was reinstated and chromium induced immunosuppression was obstructed by the EO. For the antitussive activity of EO in conscious rats, EO has been reviewed by mechanical stimulus of the air tracts tracheobronchial and laryngopharyngeal mucous region. Non-narcotic agent dropropizine was less effective in antitussive activity than that of EO but the classical narcotic antitussive drug, codeine is still at the highest efficiency for its antitussive activity. The antitussive activity shown by EO is presumed to be not only due to the antiphlogistic, anti pasmolytic and antioxidant 
potency influences but also to its effects upon the mucous secretion in the airways [30]. EO is screened for antisecretory and counterulcer behaviors, using numerous experimental models in mice, such as pylorus ligation shay rats, indometacin hypothermic stress restriction triggered stomach ulcers, and necroticants. The exhibition of antisecretory, cytoprotective, and antiulcer properties by Amla was discovered then.

\section{Memory enhancing effects}

A dose-dependent increment of Amla fine powder is observed in the memory of juvenile and old rats. It was the transposition of amnesia caused by scopolamine and diazepam. Amla Churna is displayed to be gratifying healing for the treatment of Alzheimer's disease because of its multiple advantages including memory enhancement and memory arrears [31].

\section{Management of ophthalmic conditions}

Curcuma longa, Terminalia belerica, OE, cinnamomum, ocimum sanctum, acamphora, Medespumapum and rosa damascena have been integrated into the basic concepts of various herbs; ophthacare is an herbal eye-catcher. This herbal preparation was aimed at a clinical examination of those patients with various ophthalmic afflictions such as conjunctival xerosis, conjunctivitis, acute acryocytitis, and postoperative cataracts. The herbal eye drops primarily supplied the improved treatment for eye conditions. Patients well tolerated the eye drops and no side effects were ascertained for the time of the course of study. Ophthacare has shown its beneficial function in a variety of infectious, infective, as well as degenerative ophthalmic disorders [32].

Dyslipidemia and cholesterol reduction role

Amla was investigated on low-density lipids (LDL) oxidation as well as cholesterol-induced in $\mathrm{Cu} 2+$ by cholesterol and LDL-fed rats, and its impact was examined in vitro and in vivo. For the hypercholesterolemia and prevention of atherosclerosis. Amla was concluded as an effective specimen [33]. With the flavonoids that can curtail the volumes of lipids in serum and tissues of mice induced hyperlipidemia, both EO and Mangifera are embraced, each causing breakdown and vanishing of cholesterol [34].

\section{Snake venom neutralizer}

It was the first time scrutinized that EO and vitex negundo have the anti-snake venom activity. The plant extracts were antagonized with both in vitro and in vivo models, Naja kaouthia and Vipera Rossellini venom. The V. Rossellini venom-induced coagulants, defibrinogenating hemorrhages, and inflammatory activities by these plant extracts were distinctly nullified. The strong neutralizing capacities of snake venom are seen in extracts of the plants and need even greater consideration in speculating no precipitation bands formation between snakelike venom and plant extract [35].

Antimicrobial and antimutagenicity activities

Due to its antimicrobial activities, EO has been divulged [36]. Against Escherichia coli, K.ozaenae, Klebsiella pneumonia, Proteus mirabilis, Pseudomonas aeruginosa, S. paratyphi B, and Serratia marcescens, the EO plant is endowed with puissant antibacterial activities [37]. For an antimutagenic effect with a TA 98 and TA 100 Salmonella Typhimurium Test strain, using a histidine reversal assay to test a phenobarbitone-induced hepatic s9 rat, Triphala's chloroform, water, and acetone extracts were sussed out against the direct-active mutagens, 4 nitrophenylenediamine (NPD) and sodium azide and indirect- active promoutagents, 2 Aminofluorene (2AF). The mutagenicity reticence of both direct and $s 9$ - based mutagens in the chloroform and acetone extracts in a succession was evident [38].

\section{Applications for the treatment of certain other diseases}

Triphala, which contains one of the constituents in the EO cycle, is used to medicate the ailments including such as fever, constipation, chronic ulcers, anemia, asthma and jaundice. Secluded from Triphala, polyphenolic fractions possess the anti-mutagenic effects [39]. Furthermore, the active principles of Triphala were reviewed and considered as a peerless clinical intervention for infected wounds [40]. Aqueous plant extracts have been trialed on Swiss albino mice for their radio-protective effects against sub-lethal gamma radiation ( $9 \mathrm{~Gy}$ ). The $100 \mathrm{mg} / \mathrm{kg}$ body weight against radiation discovered to be the most effective dose of fruit pulp extracts. Conspicuously, the survival time was exalted and the mortality rate of mice was lessened with this dose. Moreover, it was noticed that the loss of body weight in extract administrated irradiated animals was noticeably low as compared to animals who were given radiations only [41]. By prompting highly puissant hypolipidaemic and hypoglycemic activities maximum beneficial action is exhibited by the flavonoids derived from EO [42]. Furthermore, for elevating the hemoglobin level in rats, flavonoids are found to be very effective. Its being as anti-tumor in actions is also reported [43]. Against 
thioacetamide (TTA) and CCL4 [44], induced changes in rat liver EO fruit has been examined. Abnormal histopathology indicative of pre-fibrogenic events was caused by the treatment with TTA and CCL4 [45]. Such alteration was transposed by EO showing its preventive capability in pre-fibrogenesis of the liver [46]. The extracts of Withania are the result of a repeatable dose-dependent, colonyforming inhibition in CHO cells [47]. Hypercholesteremia is one of the key factors in the case of coronary artery disease. Besides, it has been documented that Triphala formulations show hypolipidemic properties on the experimentally induced hypercholesteremic mice [48].

\section{Conclusion}

Since ancient times Amla or Indian gooseberry played an important role in conventional medicine, tribal, and Ayurveda medicines. Several biological and biopharmaceutical studies have been performed over the last few decades in a wide group of phytochemicals such as tannins, flavonoids, terpenoids, tannins, and others, derived from Amla. Amla phytochemicals, including ellagic acid, emblicanine A, emblacani B, gallic acid, phyllantine, quercetin, phyllantidine, etc., were found to have various biological activities such as antioxidants, antimicrobials, antiinlammative, antidiabetic, anti-radiation protection, chemopreventive, healing of wounds, etc. The current research showed that some Emblica officinalis bioactive compounds are also common in other pharmaceutical plant species. Further assessment of unexplored bioactive Amla compounds is therefore required, which will expose the ever new bioactivities of this strong medicinal plant.

\section{Acknowledgment}

The authors are highly thankful to Government College University Faisalabad-Pakistan providing free full-length papers.

\section{Interest of Conflict}

The authors declare no conflict of interest.

\section{Bibliography}

1. Baliga MS and Dsouza JJ. "Amla (Emblica officinalis Gaertn), a wonder berry in the treatment and prevention of cancer". European Journal of Cancer Prevention 20.3 (2011): 225-239.

2. Sonkar N., et al. "Effect of cultivars, pretreatment and drying on physicochemical properties of Amla (Emblica officinalis) gratings". Journal of Food Science and Technology 57.3 (2020): 980-992.

3. Jain SK and Khurdiya DS. "Vitamin C enrichment of fruit juice based ready-to-serve beverages through blending of Indian gooseberry (Emblica officinalis Gaertn.) juice". Plant Foods for human nutrition 59.2 (2004): 63-66.

4. Choudhary M Grover K. "Amla (Emblica officinalis L.) Oil”. Fruit Oils: Chemistry and Functionality. Springer, Cham (2019): 875-882.

5. Habib-ur-Rehman., et al. "Studies on the chemical constituents of Phyllanthus emblica". Natural Product Research 21.9 (2007): 775-781.

6. Kumar KPS., et al. "Recent trends in potential traditional Indian herbs Emblica officinalis and its medicinal importance". Journal of Pharmacognosy and Phytochemistry 1.1 (2012): 18-28.

7. Singh E., et al. "Phytochemistry, traditional uses and cancer chemopreventive activity of Amla (Phyllanthus emblica): the sustainer". Journal of Applied Pharmacy Science 2.1 (2011): 176-183.

8. Khan KH. "Roles of Emblica officinalis in medicine-A review". Botany Research International 2.4 (2009): 218-228.

9. El-Desouky., et al. "A new cytotoxic acylated apigenin glucoside from Phyllanthus emblica L". Natural Product Research 22.1 (2008): 91-95.

10. Anjum A and Nafees H. "A Comprehensive Review on a Unani Dynamic Drug: Amla (Emblica officinalis)". Asian Plant Research Journal (2020): 1-6.

11. Veena K., et al. "The biochemical alterations following administration of Kalpaamruthaa and Semecarpus anacardium in mammary carcinoma". Chemico-biological interactions 161.1 (2006): 69-78.

12. Sancheti G., et al. "Chemopreventive action of emblica officinalis on skin carcinogenesis in mice". Asian Pacific Journal of Cancer Prevention: APJCP 6.2 (2005): 197-201.

13. Kaur S., et al. "The in vitro cytotoxic and apoptotic activity of Triphala-an Indian herbal drug". Journal of Ethnopharmacology 97.1 (2005): 15-20. 
14. Banu SM., et al. "Protective effect of Emblica officinalis ethanolic extract against 7, 12-dimethylbenz (a) anthracene (DMBA) induced genotoxicity in Swiss albino mice". Human and Experimental Toxicology 23.11 (2004): 527-531.

15. Suryanarayana P., et al. "Emblica officinalis and its enriched tannoids delay streptozotocin-induced diabetic cataract in rats". Molecular Vision 13 (2007): 1291-1297.

16. Singh MK., et al. "Anti-diabetic Effect of Emblica-officinalis (Amla) Against Arsenic Induced Metabolic Disorder in Mice”. Indian Journal of Clinical Biochemistry (2019): 1-9.

17. Bhattacharya SK., et al. "Effect of bioactive tannoid principles of Emblica officinalis on ischemia-reperfusion-induced oxidative stress in rat heart". Phytomedicine 9.2 (2002): 171-174.

18. Pramyothin P., et al. "The protective effects of Phyllanthus emblica Linn. extract on ethanol induced rat hepatic injury". Journal of Ethnopharmacology 107.3 (2006): 361-364.

19. Tasduq SA., et al. "Protective effect of a $50 \%$ hydroalcoholic fruit extract of Emblica officinalis against anti-tuberculosis drugs induced liver toxicity". Phytotherapy Research: An International Journal Devoted to Pharmacological and Toxicological Evaluation of Natural Product Derivatives 19.3 (2005): 193197.

20. Sultana S., et al. "Effect of Emblica officinalis (Gaertn) on CCl 4 induced hepatic toxicity and DNA synthesis in Wistar rats". Indian Journal of Experimental Biology 43.5 (2005): 430-436.

21. Gautam A and Shukla S. "Emblica officinalis (Amla) leaf extract potentiates antibacterial activity of some antibiotics". Journal of Pharmacognosy and Phytochemistry 6.2 (2017): 233-236.

22. Rajak S., et al. "Emblica officinalis causes myocardial adaptation and protects against oxidative stress in ischemic-reperfusion injury in rats". Phytotherapy Research: An International Journal Devoted to Pharmacological and Toxicological Evaluation of Natural Product Derivatives 18.1 (2004): 54-60.

23. Muthu PR., et al. "Amla (Emblica officinalis) improves hepatic and renal oxidative stress and the inflammatory response in hypothyroid female wistar rats fed with a high-fat diet". Jour- nal of Basic and Clinical Physiology and Pharmacology 29.2 (2018): 175-184.

24. Sairam KCHV., et al. "Antiulcerogenic effect of methanolic extract of Emblica officinalis: an experimental study". Journal of Ethnopharmacology 82.1 (2002): 1-9.

25. Dhanalakshmi S., et al. "Protective effect of Triphala on cold stress-induced behavioral and biochemical abnormalities in rats". Yakugaku Zasshi 127.11 (2007): 1863-1867.

26. Bariya AR., et al. "Assessment of Antioxidant and Sensory Properties of Amla (Emblica officinalis) Fruit and Seed Coat Powder Incorporated Cooked Goat Meat Patties". International Journal Current Microbiology Applied Science 7.7 (2018): 3306-3318.

27. Anilakumar KR Nagaraj NS and Santhanam K. "Reduction of hexachlorocyclohexane-induced oxidative stress and cytotoxicity in rat liver by Emblica officinalis gaertn". Indian Journal of Experimental Biology 45.5 (2005): 450-454.

28. Sandhya T., et al. "Protection against radiation oxidative damage in mice by Triphala". Mutation Research/Genetic Toxicology and Environmental Mutagenesis 609.1 (2006): 17-25.

29. Kumaran A and Karunakaran RJ. "Nitric oxide radical scavenging active components from Phyllanthus emblica L". Plant Foods for Human Nutrition 61.1 (2006): 1.

30. Srikumar R., et al. "Effect of Triphala on oxidative stress and on cell-mediated immune response against noise stress in rats". Molecular and Cellular Biochemistry 283.1-2 (2006): 6774.

31. Scartezzini P., et al. "Vitamin C content and antioxidant activity of the fruit and of the Ayurvedic preparation of Emblica officinalis Gaertn". Journal of Ethnopharmacology 104.1-2 (2006): 113-118.

32. Rao TP., et al. "Amla (Emblica officinalis Gaertn.) extracts reduce oxidative stress in streptozotocin-induced diabetic rats". Journal of Medicinal Food 8.3 (2005): 362-368.

33. Baliga MS., et al. "Hepatoprotective Effects of the Indian Gooseberry (Emblica officinalis Gaertn): A Revisit". Dietary Interventions in Liver Disease. Academic Press (2019): 193-201. 
34. Bhattacharya SK., et al. "Effect of bioactive tannoid principles of Emblica officinalis on ischemia-reperfusion-induced oxidative stress in rat heart". Phytomedicine 9.2 (2002): 171-174.

35. Sai RM., et al. "Cytoprotective activity of Amla (Emblica officinalis) against chromium (VI) induced oxidative injury in murine macrophages". Phytotherapy Research: An International Journal Devoted to Pharmacological and Toxicological Evaluation of Natural Product Derivatives 17.4 (2003): 430-433.

36. Nosal'ova G., et al. "Antitussive activity of the fruit extract of Emblica officinalis Gaertn. (Euphorbiaceae)". Phytomedicine 10.6-7 (2003): 583-589.

37. Al-Rehaily AJ., et al. "Gastroprotective effects of 'Amla' Emblica officinalis on in vivo test models in rats". Phytomedicine 9.6 (2002): 515-522.

38. Vasudevan M and Parle M. "Memory enhancing activity of Anwala churna (Emblica officinalis Gaertn.): An Ayurvedic preparation". Physiology and Behavior 91.1 (2007): 46-54.

39. Biswas NR., et al. "Evaluation of Ophthacare $®$ eye drops-a herbal formulation in the management of various ophthalmic disorders". Phytotherapy Research 15.7 (2001): 618-620.

40. Kim HJ., et al. "Influence of amla (Emblica officinalis Gaertn.) on hypercholesterolemia and lipid peroxidation in cholesterolfed rats". Journal of Nutritional Science and Vitaminology 51.6 (2005): 413-418.

41. Anila L and Vijayalakshmi N. "Flavonoids from Emblica officinalis and Mangifera indica-effectiveness for dyslipidemia". Journal of Ethnopharmacology 79.1 (2002): 81-87.

42. Alam MI and Gomes A. "Snake venom neutralization by Indian medicinal plants (Vitex negundo and Emblica officinalis) root extracts". Journal of Ethnopharmacology 86.1 (2003): 75-80.

43. Srikumar R., et al. "Evaluation of the growth inhibitory activities of Triphala against common bacterial isolates from HIV infected patients". Phytotherapy Research: An International Journal Devoted to Pharmacological and Toxicological Evaluation of Natural Product Derivatives 21.5 (2007): 476-480.

44. Saeed S and Tariq P. "Antibacterial activities of Emblica officinalis and Coriandrum sativum against Gram negative urinary pathogens". Pakistan Journal of Pharmaceutical Sciences 20.1 (2007): 32-35.

45. Kaur S., et al. "The in vitro antimutagenic activity of Triphala-an Indian herbal drug". Food and Chemical Toxicology 40.4 (2002): 527-534.

46. Singh DP., et al. "High-performance liquid chromatography as a tool for the chemical standardisation of Triphala-an Ayurvedic formulation". Phytochemical Analysis: An International Journal of Plant Chemical and Biochemical Techniques 19.2 (2008): 164-168.

47. Kumar MS., et al. "Triphala promotes healing of infected fullthickness dermal wound". Journal of Surgical Research 144.1 (2008): 94-101.

48. Singh I., et al. "Radioprotection of Swiss albino mice by Emblica officinalis". Phytotherapy Research: An International Journal Devoted to Pharmacological and Toxicological Evaluation of Natural Product Derivatives 19.5 (2005): 444-446.

\section{Assets from publication with us}

- Prompt Acknowledgement after receiving the article

- Thorough Double blinded peer review

- Rapid Publication

- Issue of Publication Certificate

- High visibility of your Published work

Website: www.actascientific.com/

Submit Article: www.actascientific.com/submission.php Email us: editor@actascientific.com

Contact us: +919182824667 